Ciência Florestal, Santa Maria, v. 22, n. 3, p. 505-517, jul.-set., 2012

ISSN 0103-9954

\title{
ANÁLISE ESTRUTURAL EM RELICTOS DE CERRADO NO PARQUE ESTADUAL DO GUARTELÁ, MUNICÍPIO DE TIBAGI, ESTADO DO PARANÁ, BRASIL
}

\author{
STRUCTURAL ANALYSIS IN CERRADO’S RELICTS AT STATE PARK OF GUARTELÁ, \\ TIBAGI, PARANA STATE, BRAZIL
}

\author{
Marta Regina Barrotto do Carmo ${ }^{1}$ Anna Luiza Pereira Andrade ${ }^{2}$ \\ Gisele Aparecida da S. D. dos Santos ${ }^{3}$ Marco Antônio Assis ${ }^{4}$
}

\begin{abstract}
RESUMO
O estudo foi desenvolvido em relictos de cerrado existentes no Parque Estadual do Guartelá, Tibagi-PR, $\left(24^{\circ} 39^{\prime} 10^{\prime \prime} \mathrm{S}\right.$ e $\left.50^{\circ} 15^{\prime} 25^{\prime} \mathrm{W}\right)$, os quais representam um dos poucos remanescentes extremo-meridional deste tipo de formação. Para a análise fitossociológica, foram alocadas 50 parcelas de 5 x $5 \mathrm{~m}$, incluindose os indivíduos com altura $\geq 1 \mathrm{~m}$, divididos em duas amostragens para avaliar os seguintes estratos: a) superior - plantas com DAS $\geq 3 \mathrm{~cm}$; e b) médio - plantas com DAS $<3 \mathrm{~cm}$. Ainda, em cada parcela foram estabelecidas subparcelas de $1 \times 1 \mathrm{~m}$ para amostrar o estrato inferior, constituído por indivíduos com altura $<1 \mathrm{~m}$ e $>10 \mathrm{~cm}$. No total foram amostrados 1.340 indivíduos, distribuídos em 28 famílias, 66 gêneros e 115 espécies. $\mathrm{O}$ índice de diversidade foi maior para o estrato médio $\left(H^{\prime}=3,30\right)$, seguido do superior $\left(H^{\prime}=3,09\right)$ e inferior $\left(H^{\prime}=2,91\right)$. A ocorrência frequente de moitas é uma característica notável da fisionomia das áreas do PEG, sendo que $82 \%$ das populações analisadas distribuem-se com padrão agregado. Por se tratar de uma região limítrofe de ocorrência do Cerrado, sob influência atual de um clima subtropical, mais úmido e frio, comparado à zona core de tal bioma, nota-se que estas áreas se caracterizam por uma diminuição na estatura, riqueza e diversidade de sua flora. Apesar disso, preserva espécies características dos cerrados brasileiros, mas, por apresentarem distribuição em relictos e estes terem sido em parte devastados, algumas estão incluídas na lista vermelha de plantas ameaçadas de extinção no Estado do Paraná, o que evidencia a importância da preservação e manejo destas áreas.
\end{abstract}

Palavras-chave: Cerrado Meridional; estrutura de comunidade; Campos Gerais do Paraná.

\begin{abstract}
This study was accomplished in existing Cerrado's relicts at State Park Guartelá, Tibagi, PR (2439'10'S and $50^{\circ} 15^{\prime} 25^{\prime}$ 'W), which represent one of the last extreme-meridional remanescents of this type of formation. Fifty plots with dimensions of $5 \times 5 \mathrm{~m}$ were allocated for phyto-sociological analysis, including in the sample individuals with height $\geq 1 \mathrm{~m}$, divided into two samples to evaluate the following strata: a) upper- plants with DAS $\geq 3 \mathrm{~cm}$, and b) intermediate - plants with DAS $<3 \mathrm{~cm}$. Moreover, in each plot, there were established sub-plots with dimensions of $1 \mathrm{x} 1 \mathrm{~m}$ for sampling the lower stratum, comprised by individuals with height $<1 \mathrm{~m}$ and $>10 \mathrm{~cm}$. The complete survey found 1340 individuals distributed in 28 families, 66 genera and 115 species. The diversity index was higher for the mean component $\left(H^{\prime}=3.30\right)$, followed by the superior component $\left(\mathrm{H}^{\prime}=3.09\right)$ and the inferior $\left(\mathrm{H}^{\prime}=2.91\right)$. The frequent occurrence of bushes is a remarkable characteristic of the physiognomy of the areas inside the park and $82 \%$ of the populations studied are distributed in aggregated standard. Because it is a marginal region of occurrence of
\end{abstract}

1. Bióloga, Dra , Professora Adjunta do Departamento Biologia Geral, Universidade Estadual de Ponta Grossa, Av. Carlos Cavalcanti, 4748, Uvaranas, CEP 84030-910, Ponta Grossa (PR). mrcarmo@uepg.br

2. Bióloga, Msc., Doutoranda do Programa de Pós-Graduação em Engenharia Florestal, Universidade Federal do Paraná, Av. Pref. Lothário Meissner, 900, Jardim Botânico, CEP 80210-170, Curitiba (PR). annnaluiza@yahoo.com.br

3. Bióloga, Mestre em Ciências Biológicas pela Universidade Estadual de Londrina, Rodovia Celso Garcia Cid, Campus Universitário, Caixa Postal 6001, CEP 86051-980, Londrina (PR). gidoratti@hotmail.com

4. Biólogo, Dr., Professor Assistente da Universidade Estadual Paulista, campus de Rio Claro, Av. 24 A, 1515, Bela Vista, CEP 13506-900, Rio Claro (SP). massis@rc.unesp.br

Recebido para publicação em 06/07/2010 e aceito em 26/05/2011

Ci. Fl., v. 22, n. 3, jul.-set., 2012 
cerrado vegetation, now under the influence of a subtropical climate, more humid and cold, compared to the core area of this biome, it is noted that these areas are characterized by a decrease in stature, richness and diversity of its flora. Nevertheless, they preserve typical species of the Brazilian Savannah; but, due to their distribution in relicts and been, in part, devastated, some are included in the red list of endangered plants in Parana state, which evidences the importance of conservation and management of these areas.

Keywords: Southern "Cerrado", Brazilian savannah; community structure; Campos Gerais do Paraná.

\section{INTRODUÇÃO}

Constituindo a segunda maior formação vegetal brasileira, o Cerrado abrange principalmente uma área contínua localizada no planalto Central (RATTER et al., 1997), sendo caracterizada por um clima estacional (VELOSO et al., 1991). No entanto, ocorre também como área disjunta nas regiões Norte, Nordeste e Sul do Brasil (EITEN, 1972; RIBEIRO e WALTER, 1998), em meio a outras paisagens dominantes com variações climáticas distintas da área core.

Muitas destas áreas disjuntas têm revelado uma menor ocorrência de espécies endêmicas e até mesmo uma diminuição da riqueza florística (SANAIOTTI et al., 1997; RATTER et al., 1997; UHLMANN et al., 1998; COSTA et al., 2004), quando comparadas com a região nuclear. Assim, nota-se uma tendência das características típicas dos cerrados irem se atenuando ou até desaparecendo à medida que se expande das áreas core para áreas periféricas (PASSOS, 2003).

Dentre as regiões marginais de Cerrado, o limite austral de sua ocorrência localiza-se no Estado do Paraná (UHLMANN et al., 1998; TOREZAN, 2002), onde aparece como ilhas esparsas (HUECK, 1972), sendo consideradas relictas de uma vegetação que dominou o estado em épocas pretéritas (MAACK, 1981), quando havia um clima semiárido do Quaternário Antigo, estando em discordância com o clima atual, mais úmido (MAACK, 1949). Estima-se que as áreas de cerrado paranaense cobriam originalmente cerca de $1.882 \mathrm{Km}^{2}$ (MAACK, 1981), o que correspondia aproximadamente a $1 \%$ do Estado.

Apesar de vários pesquisadores terem concentrado esforços para o conhecimento da flora do Cerrado em diferentes regiões do Brasil (RATTER et al., 2003), poucos trabalhos botânicos deram ênfase à composição (TAKEDA et al., 1996; VON LINSINGEN et al., 2006), e estrutura (UHLMANN et al., 1997; 1998) dos remanescentes paranaenses, o que parecem insuficientes para uma melhor caracterização da vegetação no extremomeridional do bioma, necessitando de estudos para ampliar seu entendimento. Além disso, há um consenso que extensas áreas de cerrado estão sendo perdidas sem um conhecimento detalhado dos aspectos florísticos e fitossociológicos, e no Paraná se agrava ainda mais, já que as áreas são de dimensões restritas e muitas foram descaracterizadas, ou até mesmo totalmente destruídas (PARANÁ, 1995), sendo que dentre as plantas ameaçadas de extinção no estado, 10,3\% têm ocorrência nos relictos de cerrado (PARANÁ, 1995).

A proposta do presente trabalho foi realizar uma caracterização qualitativa e quantitativa em áreas de cerrado dentro do Parque Estadual do Guartelá (Tibagi, Paraná), com intuito de responder as seguintes questões: a) Como se caracteriza a estrutura fitossociológica dos cerrados em fragmentos reduzidos de áreas relictas, tendo em vista que essa organização ocorre sob condições ambientais próprias? b) São mantidas a composição florística típica e a diversidade dos cerrados de outras regiões?

\section{MATERIAL E MÉTODOS}

Totalizando uma área de 798,97 ha, o Parque Estadual do Guartelá (PEG), está inserido na Área de Proteção Ambiental (APA) da Escarpa Devoniana e localiza-se no Município de Tibagi, Estado do Paraná, Sul do Brasil, nas coordenadas $24^{\circ} 39^{\prime} 10^{\prime \prime} \mathrm{S}$ e $50^{\circ} 15^{\prime} 25^{\prime \prime} \mathrm{W}$. O parque caracterizase por um predomínio de fisionomias campestres (Estepe gramíneo-lenhosa, Estepe higrófila e Refúgios Vegetacionais Rupestres) e distribuição em mosaico de floresta com Araucária (Floresta Ombrófila Mista) e de cerrado (Savana Arborizada) (VELOSO et al., 1991).

O PEG apresenta um relevo com topografia muito diversificada, variando de suave-ondulado a extremamente acidentado, com as escarpas chegando a mais de $100 \mathrm{~m}$ de altura (DIEDRICHS, 
1995), sendo que no limite Leste encontra-se o rio Iapó, o qual se situa na porção central do canyon do Guartelá, este se estende por cerca de $30 \mathrm{~km}$ entre os municípios de Castro e Tibagi (PR) e com desnível máximo de $450 \mathrm{~m}$ (MELO, 2002).

De um modo geral, os solos da região são pouco profundos, predominando os Cambissolos e os Litossolos (Neossolos Litólicos) constituídos de areia esbranquiçada, proveniente da decomposição do arenito Furnas, muito resistente aos fatores de diagênese, sendo também comuns os afloramentos rochosos (KLEIN e HATSCHBACH, 1970/71).

O clima da região, de acordo com o sistema de classificação de Köeppen, é do tipo Cfa (úmido subtropical) com influência direta do clima $\mathrm{Cfb}$ (úmido temperado), apresentando temperaturas médias em torno de $18{ }^{\circ} \mathrm{C}$, sendo as médias máximas de $23{ }^{\circ} \mathrm{C}$ e mínimas de $14{ }^{\circ} \mathrm{C}$ (IAP, 2002; CRUZ, 2007). As chuvas são bem distribuídas, ao longo do ano, na região, a precipitação média anual encontra-se entre 1.400 e $1.600 \mathrm{~mm}$, sendo que a umidade relativa anual permanece entre $80 \mathrm{e}$ $85 \%$, porém, há um período chuvoso ligeiramente acentuado de outubro a março (CRUZ, 2007).

$\mathrm{O}$ estudo foi desenvolvido nas três pequenas manchas de cerrado presentes no PEG e que somam 11,24 ha. Estas áreas localizam-se na região noroeste do parque, em meio à paisagem campestre e florestal, caracterizadas por uma vegetação predominantemente arbustiva, com ocorrência de árvores e arvoretas esparsas.

Para a amostragem foram alocadas 50 parcelas de $5 \times 5 \mathrm{~m}\left(25 \mathrm{~m}^{2}\right)$, distribuídas de maneira sistematizada, duas a duas, com espaçamento de $20 \mathrm{~m}$ entre elas, abrangendo três fragmentos (14, 16 e 20 parcelas em cada fragmento). Todos os indivíduos que apresentavam altura igual ou superior a $1 \mathrm{~m}$ foram registrados, porém, dividiu-se em dois estratos de amostragens para possibilitar a comparação com outros trabalhos em áreas de cerrado: a) estrato superior (ES) plantas com diâmetro a altura do solo (DAS) igual ou superior a $3 \mathrm{~cm}$, incluindo os indivíduos com caule perfilhado que, na somatória das medidas, ultrapassasse este valor; e b) estrato médio (EM) plantas com DAS menor que $3 \mathrm{~cm}$. Ainda, em cada parcela, foram estabelecidas subparcelas de 1 x 1 m para amostrar o estrato inferior (EI), constituído por indivíduos com altura inferior a $1 \mathrm{~m}$ e superior a $10 \mathrm{~cm}$, excluindo-se formas graminosas.

Para todos os indivíduos amostrados foram registradas medidas do diâmetro e altura. Também foram realizadas coletas para a identificação dos espécimes, sendo que exemplares férteis, após herborização, foram incluídos no Herbário HUPG. O sistema de classificação utilizado para composição das famílias botânicas foi o APG II, Angiosperm Phylogeny Group (2003).

A partir dos dados levantados em campo, calculou-se para cada classe de amostragem (superior, médio e inferior), utilizando o programa Fitopac (SHEPHERD, 1995), os descritores fitossociológicos: densidade, dominância e frequência relativas (MUELLER-DOMBOIS e ELLENBERG, 1974); além da diversidade de Shannon (H'). Relações de similaridade foram calculadas através do índice de Sorensen (MUELLER-DOMBOIS e ELLENBERG, 1974).

Para a avaliação da distribuição espacial das populações, foram selecionadas aquelas espécies representadas por cinco ou mais indivíduos amostrados nos diferentes estratos. Para análise, empregou-se o Coeficiente de Dispersão (CD), obtido pela razão variância/ média (BROWER e ZAR, 1984). Valor de CD igual a 1, indica um padrão aleatório; $C D$ menor que 1 , o padrão é uniforme; e CD maior que 1 , representa uma distribuição agregada. Para testar se os valores do CD diferiam significativamente da unidade, utilizou-se o teste T (BROWER e ZAR, 1984).

Para a verificação da distribuição geográfica de árvores e arbustos amostrados neste estudo, foram incluídas na análise apenas aquelas espécies que tiveram identificação em nível específico (as quais somaram 50 espécies). Tal comparação foi realizada através de consultas a listagens florísticas de trabalhos localizados em cerrados, considerando-se os seguintes grupos fitogeográficos reconhecidos por Ratter et al. (2003): a) Meridional (DURIGAN et al., 1994, 1999, 2002; BATALHA et al., 1997; UHLMANN et al., 1998; ARAÚJO et al., 1999; BATALHA e MANTOVANI, 2000, 2001; FIDELIS e GODOY, 2003); b) Centro-Sudeste (MEIRA NETO e SAPORETTI JÚNIOR, 2002; ANDRADE et al., 2002; FONSECA e SILVA JUNIOR, 2004; ASSUNÇÃO e FELFILI, 2004); c) Centro-Oeste (NASCIMENTO e SADDI, 1992; SILVA et al., 2002; FELFILI et al., 2002); d) Norte-Nordeste (BARREIRA et al., 2002; COSTA et al., 2004); e) em áreas isoladas da Amazônia (EGLER, 1960; BASTOS, 1984; MIRANDA, 1993; SANAIOTTI et al., 1997). 


\section{RESULTADOS E DISCUSSÃO}

O levantamento total amostrou 1.340 indivíduos, distribuídos em 28 famílias, 66 gêneros e 115 espécies, sendo que destas, seis amostras ainda não foram identificadas.

Comparando as três classes de amostragem, notou-se que existe um aumento gradativo na densidade total dos indivíduos e no número de espécies do estrato superior $(\mathrm{DT}=2376 ; \mathrm{Nsp}=47)$ para o médio $(\mathrm{DT}=2792 ; \mathrm{Nsp}=60)$, atingindo os maiores valores no inferior $(\mathrm{DT}=5560$; $\mathrm{Nsp}=76$ ). Esta característica parece ocorrer em áreas de Cerrado sensu stricto, já que levantamentos florísticos têm relatado que a proporção do número de espécies do estrato herbáceo é maior que o arbustivo-arbóreo (MANTOVANI e MARTINS, 1993; BATALHA e MANTOVANI, 2000). No entanto, o índice de diversidade foi maior para o estrato médio $\left(\mathrm{H}^{\prime}=3,30\right)$, seguido do superior $\left(H^{\prime}=3,09\right)$ e do inferior $\left(H^{\prime}=2,91\right)$. Isto revela que, apesar do maior número de espécie compondo o estrato inferior, há uma desigualdade acentuada na distribuição dos indivíduos, acarretando em menor heterogeneidade.

Os estratos superior e médio tiveram uma similaridade de Sorensen de $63 \%$ que, por sua vez, alcançaram em torno de $44 \%$, igualmente para o estrato inferior. Estes índices mostraram a semelhança florística nos dois estratos de amostragem, com inclusão de plantas lenhosas e que está ocorrendo uma regeneração satisfatória destas espécies no estrato herbáceo. No entanto, $54 \%$ das espécies do estrato inferior são exclusivas, já que são tipicamente ervas deste estrato.

Em todos os estratos avaliados, a família Myrtaceae obteve a maior riqueza florística (Figura 1). Observa-se que as nove famílias mais representativas corresponderam a $72 \%$ do total de espécies encontradas no estrato superior, $82 \%$ para o estrato médio e $70 \%$ para o estrato inferior, tendo este último uma riqueza maior de Fabaceae, Malpighiaceae e Rubiaceae, quando comparado com os demais estratos (Figura 1). Estas nove famílias têm uma distribuição ampla nos cerrados brasileiros (SILVA et al., 2002; MEIRA NETO e SAPORETTI JUNIOR, 2002; BARREIRA et al., 2002; FELFILI et al., 2002; FONSECA e SILVA JUNIOR, 2004; PINTO et al., 2009), inclusive para os estratos inferior e médio, como relatado por Mantovani e Martins (1993), Batalha et al. (1997), Durigan et al. (1999) e Batalha e Mantovani (2001).

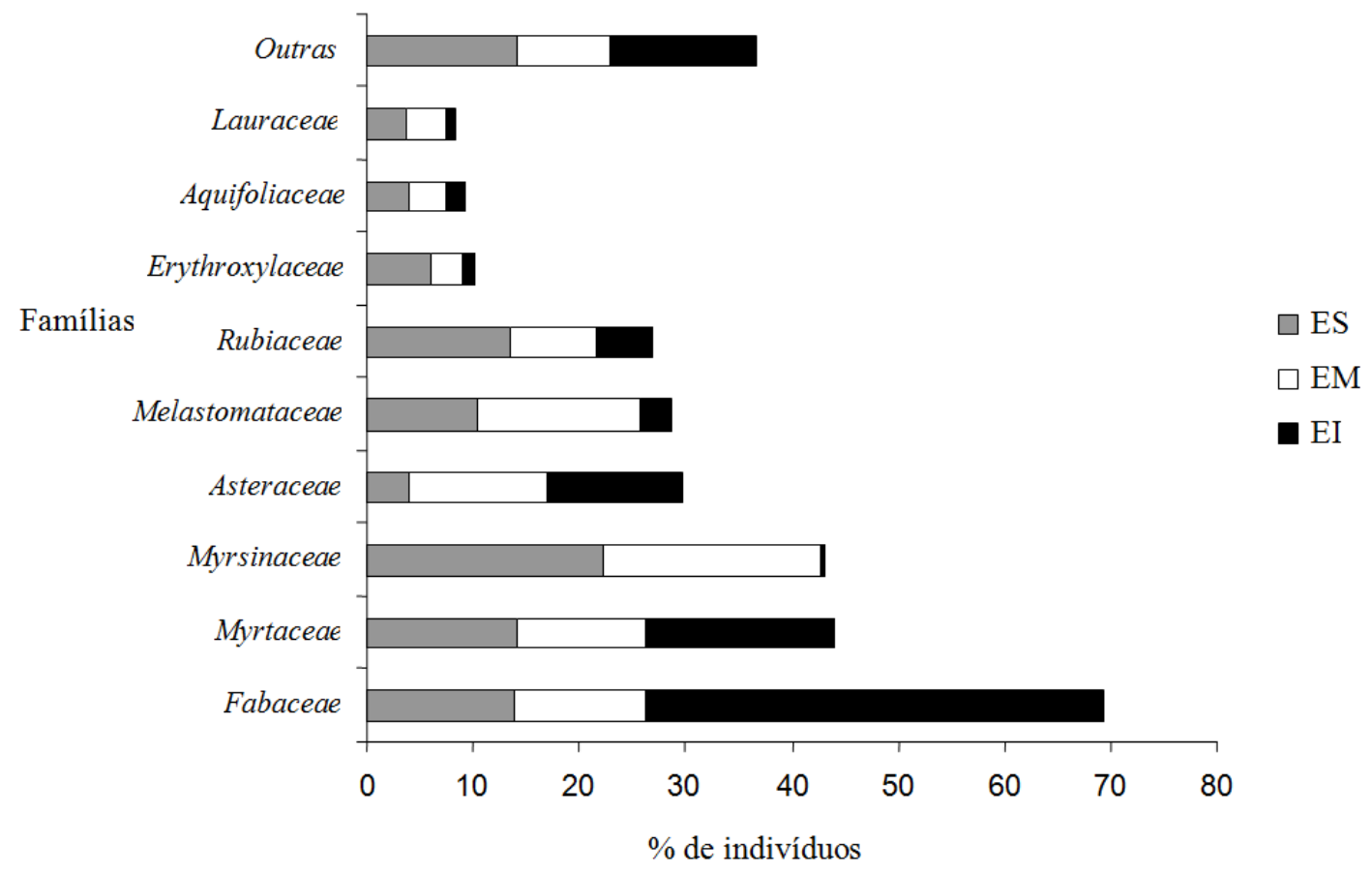

FIGURA 1: Porcentagem de indivíduos por famílias ocorrentes nas áreas de cerrado do Parque Estadual do Guartelá, Tibagi-PR. ES - estrato superior; EM - estrato médio; EI - estrato inferior.

FIGURE 1: Percentage of individuals in families occurring in cerrado Guartelá State Park, Tibagi-Paraná state. ES- upper stratum, EM- middle stratum, EI- understory. 
No entanto, Vochysiaceae, considerada uma das famílias mais importantes deste bioma pelos altos valores de biomassa (FELFILI et al., 2002; SILVA et al., 2002; ANDRADE et al., 2002; PINTO et al., 2009) no cerrado do PEG, apresentou baixa densidade ( $\mathrm{DR}=0,37 \%)$, com ocorrência apenas de Qualea cordata. A baixa ocorrência de espécies desta família parece ser uma característica dos cerrados paranaenses, já que Uhlmann et al. (1998) também relataram pouca representatividade para o Parque Estadual do Cerrado em Jaguariaíva (PR).

Para o estrato superior (Tabela 1), as espécies Myrsine umbellata, Miconia sellowiana, Ouratea spectabilis, Plenckia populnea, Myrcengenia alpigena e Alibertia concolor obtiveram os maiores valores de dominância relativa, representando conjuntamente $58 \%$ do total. $\mathrm{O}$ efeito foi ocasionado pelos altos valores de área basal, resultado de indivíduos arbóreos (Ouratea spectabilis e Plenckia populnea), ou de arvoretas perfilhadas (Myrsine umbellata, Miconia sellowiana, Myrcengenia alpigena e Alibertia concolor) que ocorrem em grandes touceiras, caracterizando a fisionomia da área de estudo.

Myrsine umbellata foi a espécie que obteve o maior valor de importância, representando $22 \%$ dos indivíduos amostrados. Alibertia concolor com grande frequência, obteve a segunda posição em valor de importância, seguida de Miconia sellowiana. Além destas espécies, também mereceram destaque pelos altos valores de importância (VI): Myrceugenia alpigena, Ouratea spectabilis, Plenckia populnea, Erythroxylum suberosum, Copaifera langsdorffii, Calyptranthes concinna e Myrciaria cuspidata (Tabela 1). Estas dez espécies representaram $63 \%$ do VI total e caracterizaram a área de estudo.

Dados estruturais de áreas de cerrado, levando-se em conta o critério de inclusão entre 3 e $5 \mathrm{~cm}$ para o diâmetro basal, medidos até $50 \mathrm{~cm}$ de altura, mostram uma alta variação nos valores de diversidade, número de espécies e densidade total (Tabela 2). Os resultados comparativos para as duas áreas estudadas no Paraná (UHLMANN et al., 1998), revelaram que o cerrado do PEG obteve valores de diversidade e número de espécies superiores aos encontrados para o Parque Estadual do Cerrado. Possivelmente esta diferença está influenciada pelo critério de amostragem, pois o método do presente trabalho permitiu registrar um maior número de espécies, já que Uhlmann et al. (1998) incluíram os indivíduos que tivessem ao menos um perfilho com diâmetro basal maior que $3 \mathrm{~cm}$, priorizando as de maior porte, diferente deste estudo, que incluiu os indivíduos com caule perfilhado que, na somatória das medidas, ultrapassasse este valor. Estes autores admitiram que a amostragem da flora lenhosa foi subestimada em razão da escolha do critério, excluindo muitas espécies de pequeno porte. A diferença metodológica acarretou, ainda, em um maior número de indivíduos amostrados no PEG e, consequentemente, em uma maior densidade total (Tabela 2).

Em relação às características estruturais de áreas de cerrado do Distrito Federal e Goiás (Tabela 2), nota-se uma vegetação lenhosa mais rica, melhor distribuída e com maior diversidade, quando comparadas com a área do presente estudo, mesmo com restrições nos limites de inclusão de indivíduos (Diâmetro Basal $\geq 5 \mathrm{~cm}$, medido a 30 $\mathrm{cm}$ do solo). A riqueza e diversidade dos cerrados paranaenses também são menores em relação aos paulistas e mineiros (Tabela 2), apesar de exceções nos Municípios de Brotas (SP) (DURIGAN et al., 2002) e Brasilândia (MG) (BARREIRA et al., 2002), tendo em vista a restrição maior na amostragem destes levantamentos. Porém, supera os valores de algumas áreas do Mato Grosso e da Amazônia (Tabela 2), possivelmente, em função de diferenças nos critérios de inclusão. Apesar dos diferentes critérios de inclusão, nota-se que existe uma tendência da diversidade presente na região nuclear do cerrado ir diminuindo à medida que, geograficamente, as áreas vão se tornando distantes, com condições climáticas diferentes. Para o estrato superior, foram encontrados $2,3 \mathrm{~m}$ de altura média e $7,5 \mathrm{~cm}$ de diâmetro médio. Comparando estes valores com aqueles encontrados por Uhlmann et al. (1998) para o Parque Estadual do Cerrado-PR (alt. média $=2,96 \mathrm{~m}$ e diâm. médio $=9,49 \mathrm{~cm}$ ), César et al. (1988) para Corumbataí-SP (alt. média $=4,22 \mathrm{~m}$ e diâm. médio $=9,66 \mathrm{~cm}$ ), Silva et al. (2002) para Caldas Novas-GO (alt. médias $=2,52 \mathrm{~m}$ $-3,04$ m e diâm. médios $=9,49 \mathrm{~cm}-9,29 \mathrm{~cm})$, temse uma vegetação com médias de altura e diâmetro com valores menores, caracterizando um cerrado com indivíduos de baixa estatura. As espécies com alturas máximas, acima de $4 \mathrm{~m}$, somaram apenas 6,1\%, sendo caracterizada por Stryphnodendron adstringens, as demais espécies foram: Myrsine umbellata, Plenckia populnea, Myrciaria ciliolata, Ouratea spectabilis, Copaifera langsdorffii e Tabebuia aurea. 
TABELA 1: Dados estruturais das espécies ocorrentes nos estratos superior (ES), médio (EM) e inferior (EI), amostradas no cerrado do Parque Estadual do Guartelá, Município de Tibagi-PR.

TABLE 1: Structural data of species in upper stratum (ES), middle stratum (EM) and understory (EI) sampled in cerrado Guartelá State Park, Tibagi, Paraná state.

\begin{tabular}{|c|c|c|c|c|c|c|c|c|}
\hline \multirow{2}{*}{ Espécies } & \multicolumn{3}{|c|}{ NI } & \multicolumn{3}{|c|}{ VI } & \multirow{2}{*}{$\mathrm{CD}$} & \multirow{2}{*}{ GF } \\
\hline & ES & EM & EI & ES & EM & EI & & \\
\hline Myrsine umbellata Mart. & 066 & 071 & 004 & 50,81 & 55,13 & 4,33 & $13,22 b$ & 1 \\
\hline Alibertia concolor (Cham.) K. Schum. & 040 & 028 & 017 & 28,62 & 18,94 & 6,58 & $5,96 \mathrm{~b}$ & 1 \\
\hline Miconia sellowiana Naudin & 021 & 022 & 001 & 22,38 & 19,63 & 3,92 & $6,22 \mathrm{~b}$ & 1 \\
\hline Myrceugenia alpigena (O. Berg) Land. & 017 & 003 & 002 & 20,95 & 3,20 & 2,92 & $1,13 \mathrm{a}$ & 6 \\
\hline Ouratea spectabilis (Mart. ex Engl.)Engl. & 008 & 001 & 002 & 16,11 & 1,71 & 2,36 & $1,54 \mathrm{~b}$ & $1,2,3$ \\
\hline Erythroxylum suberosum St. Hill. & 010 & 002 & 005 & 11,16 & 2,97 & 3,16 & $3,07 \mathrm{~b}$ & $1,2,3,4$ \\
\hline Copaifera langsdorffii Desf. & 009 & 005 & 003 & 10,20 & 4,35 & 2,73 & $1,99 \mathrm{~b}$ & 1,2 \\
\hline Calyptranthes concinna DC. & 010 & 003 & 002 & 9,90 & 3,15 & 0,76 & $2,21 \mathrm{~b}$ & 1 \\
\hline Myrciaria cuspidata $\mathrm{O}$. Berg & 005 & 002 & 043 & 7,70 & 1,99 & 12,02 & $24,78 \mathrm{~b}$ & 6 \\
\hline Ilex sp1 & 012 & 012 & 012 & 7,54 & 11,03 & 11,21 & $6,07 \mathrm{~b}$ & - \\
\hline Leandra petropolitana Cogn. & 007 & 019 & 006 & 6,24 & 14,89 & 1,63 & $11,21 \mathrm{~b}$ & 6 \\
\hline Periandra mediterranea (Vell.) Taub. & 004 & 014 & 284 & 2,84 & 11,16 & 90,28 & $7,56 \mathrm{~b}$ & - \\
\hline Ocotea tristis (Nees) Mez & 003 & 003 & 006 & 2,83 & 3,28 & 2,95 & $2,95 \mathrm{~b}$ & 6 \\
\hline Erythroxylum deciduum A. St.-Hil. & 003 & 003 & 004 & 2,73 & 4,12 & 5,70 & $1,22 \mathrm{a}$ & $1,2,4$ \\
\hline Byrsonima brachybotrya Nied. & 002 & 002 & 002 & 2,12 & 1,79 & 2,51 & $1,24 \mathrm{a}$ & 1 \\
\hline Myrcia multiflora Lam. DC. & 002 & 007 & 002 & 2,05 & 6,54 & 1,52 & $2,14 \mathrm{~b}$ & $1,2,5$ \\
\hline Mimosa lanata Benth. & 002 & 020 & 001 & 1,96 & 12,21 & 0,63 & $5,17 \mathrm{~b}$ & 6 \\
\hline Caryocar brasiliensis Cambess. & 001 & 001 & 007 & 1,19 & 1,71 & 6,63 & $4,14 \mathrm{~b}$ & $1,2,4$ \\
\hline Psidium cinereum Mart. & 001 & 003 & 002 & 1,06 & 3,00 & 2,38 & $1,24 \mathrm{a}$ & 1 \\
\hline Eugenia bimarginata DC. & 001 & 016 & 013 & 1,06 & 12,44 & 5,10 & $4,76 \mathrm{~b}$ & 1,2 \\
\hline Campomanesia pubescens ( DC.) O. Berg. & 001 & 004 & 014 & 1,05 & 3,98 & 5,71 & $2,97 \mathrm{~b}$ & 1 \\
\hline Myrcia venulosa $\mathrm{DC}$ & 001 & 001 & 004 & 1,00 & 1,12 & 1,15 & $3,28 \mathrm{~b}$ & 1 \\
\hline Baccharis dracunculifolia DC. & 007 & 010 & - & 6,59 & 10,75 & - & $2,11 \mathrm{~b}$ & 1,2 \\
\hline Symplocos tenuifolia Brand & 006 & 005 & - & 6,31 & 5,60 & - & $3,06 \mathrm{~b}$ & 1 \\
\hline Cinnamonum sellowianum (Nees) Kost. & 006 & 008 & - & 5,90 & 8,76 & - & $2,48 \mathrm{~b}$ & 1 \\
\hline Pera glabrata (Schott) Baill. & 004 & 003 & - & 3,90 & 2,29 & - & $3,79 \mathrm{~b}$ & 1 \\
\hline Gochnatia argyrea (Dusen ex Malm) Cabr. & 004 & 006 & - & 3,61 & 6,10 & - & $1,72 b$ & 6 \\
\hline Erythroxylum buxus Peyr. & 003 & 001 & - & 3,01 & 0,97 & - & - & 1 \\
\hline Erythroxylum cuneifolium (Mart.) Schulz & 002 & 003 & - & 2,31 & 2,31 & - & $2,14 \mathrm{~b}$ & 1 \\
\hline Tabebuia aurea (Manso) Benth \& Hooker & 002 & 002 & - & 2,26 & 2,23 & - & $1,33 \mathrm{a}$ & $1,2,3,4$ \\
\hline Aegiphila sp. 2 & 002 & 001 & - & 2,19 & 0,99 & - & - & - \\
\hline Trembleya parviflora (Don) Cogn. & 001 & 001 & - & 1,10 & 0,92 & - & - & 1 \\
\hline Calea sp. & 001 & 001 & - & 1,09 & 0,83 & - & - & - \\
\hline Plenckia populnea Reissek & 004 & - & 016 & 11,70 & - & 15,66 & $1,53 \mathrm{~b}$ & $1,2,3$ \\
\hline Myrcia arborescens O. Berg. & 003 & - & 003 & 6,10 & - & 6,12 & $2,97 \mathrm{~b}$ & 6 \\
\hline Stryphnodendron adstringens Mart.Coville & 003 & - & 002 & 3,93 & - & 0,91 & $1,33 \mathrm{a}$ & $1,2,3$ \\
\hline Myrcia breviramis (O. Berg ) D. Legrand & 001 & - & 002 & 2,51 & - & 0,80 & - & 1 \\
\hline Casearia sylvestris $\mathrm{Sw}$. & 001 & - & 004 & 1,22 & - & 5,04 & $2,14 \mathrm{~b}$ & $1,2,3,4$ \\
\hline Acosmium subelegans (Mohl.) Yakovlev & 005 & - & - & 6,11 & - & - & $1,33 \mathrm{a}$ & 1,2 \\
\hline
\end{tabular}

Continua ... 
TABELA 1: Continuação ...

TABLE 1: Continued ...

\begin{tabular}{|c|c|c|c|c|c|c|c|c|}
\hline \multirow{2}{*}{ Espécies } & \multicolumn{3}{|c|}{ NI } & \multicolumn{3}{|c|}{ VI } & \multirow{2}{*}{$\mathrm{CD}$} & \multirow{2}{*}{ GF } \\
\hline & ES & EM & EI & ES & EM & EI & & \\
\hline Qualea cordata Spreng. & 005 & - & - & 4,80 & - & - & $3,37 \mathrm{~b}$ & 1,2 \\
\hline Symplocos pubescens Klotzch \& Benth. & 002 & - & - & 2,63 & - & - & - & 1 \\
\hline Miconia albicans Sw. Triana & 002 & - & - & 2,20 & - & - & - & $1,2,3,5$ \\
\hline Alchornea triplinervia (Spreng.)Müll. Arg. & 002 & - & - & 2,14 & - & - & - & 1 \\
\hline Ocotea pulchella (Nees) Mez & 002 & - & - & 1,83 & - & - & - & 1 \\
\hline Aegiphila sp. 1 & 001 & - & - & 1,73 & - & - & - & - \\
\hline Didimopanax sp. & 001 & - & - & 1,70 & - & - & - & - \\
\hline $\begin{array}{l}\text { Handroanthus chrysotrichus (Mart ex DC) } \\
\text { Mattos1 }\end{array}$ & 001 & - & - & 1,62 & - & - & - & 2 \\
\hline Symphyopappus cuneatus (DC)Sch ex Baker & - & 013 & 003 & - & 12,04 & 1,47 & $1,80 \mathrm{~b}$ & - \\
\hline Croton serpyllifolius Baill. & - & 010 & 006 & - & 8,37 & 3,89 & $1,08 \mathrm{a}$ & - \\
\hline Miconia cinerascens Miq. & - & 004 & 010 & - & 4,48 & 3,77 & $6,13 \mathrm{~b}$ & 6 \\
\hline Tibouchina sp. & - & 003 & 001 & - & 2,30 & 0,66 & - & - \\
\hline Lantana $\mathrm{sp}$ & - & 001 & 014 & & 1,25 & 3,08 & $11,33 b$ & - \\
\hline Banisteriopsis campestris (A.Juss.) Little & - & 001 & 002 & - & 1,05 & 1,46 & - & 1,2 \\
\hline Calibrachoa ericifolia (R.E.Fr.) Wijs. & - & 001 & 001 & - & 0,84 & 0,64 & - & - \\
\hline Gochnatia polymorpha (Less.) Cabrera & - & 005 & - & - & 4,92 & - & $2,14 \mathrm{~b}$ & 1 \\
\hline Calea parviflora Baker & - & 004 & - & - & 2,70 & - & - & - \\
\hline Persea sp. & - & 002 & - & - & 2,48 & - & - & - \\
\hline Lithraea molleoides (Vell.) Engl. & & 002 & & & 2,43 & & - & 1 \\
\hline Vernonia mollissima D. Don. & - & 002 & - & - & 1,74 & - & - & - \\
\hline Calea hispida (DC.) Baker & - & 002 & - & - & 1,73 & - & - & - \\
\hline $\begin{array}{l}\text { Chamaecrista punctata (Vog.) Irwin \& } \\
\text { Barneby }\end{array}$ & - & 002 & - & - & 1,49 & - & - & - \\
\hline Myrcia rostrata $\mathrm{DC}$ & - & 001 & - & - & 1,12 & - & - & $1,2,5$ \\
\hline Melastomataceae sp1 & - & 001 & - & - & 1,09 & - & - & - \\
\hline Miconia sp. & - & 001 & - & - & 1,03 & - & - & - \\
\hline Erythroxylum campestris A. St.-Hil. & - & 001 & - & - & 0,99 & - & - & $1,2,3$ \\
\hline Baccharis calvescens DC. & - & 001 & - & - & 0,92 & - & - & - \\
\hline Leandra regnelli (Triana) Cogn. & - & 001 & - & - & 0,92 & - & - & 6 \\
\hline Aureliana sp. & - & 001 & - & - & 0,91 & - & - & - \\
\hline Myrcia floribunda Miq. & - & 001 & - & - & 0,90 & - & - & 6 \\
\hline Psidium rufum Mart. ex DC. & - & 001 & - & - & 0,90 & - & - & - \\
\hline Tibouchina hatschbachii Wurdack & - & 001 & - & - & 0,86 & - & - & - \\
\hline Asteraceae sp. 2 & - & 001 & - & - & 0,86 & - & - & - \\
\hline $\begin{array}{l}\text { Chamaecrista ramosa (Irwin) Irwin \& } \\
\text { Barneby }\end{array}$ & - & 001 & - & - & 0,84 & - & - & - \\
\hline Baccharis semiserrata (Steud.) G. Barroso & - & - & 021 & - & - & 9,28 & $2,06 \mathrm{~b}$ & - \\
\hline Eupatorium multifidum DC. & - & - & 023 & - & - & 7,75 & $3,66 \mathrm{~b}$ & - \\
\hline Eugenia pitanga (O. Berg) Kiaersk. & - & - & 029 & - & - & 7,26 & $7,54 \mathrm{~b}$ & 1 \\
\hline Palicourea rigida Kunth & - & - & 013 & - & - & 4,50 & $7,35 b$ & - \\
\hline
\end{tabular}

Continua ... 
TABELA 1: Continuação ...

TABLE 1: Continued ...

\begin{tabular}{|c|c|c|c|c|c|c|c|c|}
\hline \multirow{2}{*}{ Espécies } & \multicolumn{3}{|c|}{ NI } & \multicolumn{3}{|c|}{ VI } & \multirow{2}{*}{$\mathrm{CD}$} & \multirow{2}{*}{ GF } \\
\hline & ES & EM & EI & ES & EM & EI & & \\
\hline Orthopappus angustifolius (Swartz) Gleason & - & - & 014 & - & - & 3,98 & $4,09 \mathrm{~b}$ & - \\
\hline Vernonia nudiflora Less. & - & - & 003 & - & - & 3,40 & - & - \\
\hline Hexachlamys hamiltonii Mattos & - & - & 005 & - & - & 2,86 & $1,33 \mathrm{a}$ & - \\
\hline Indeterminada 3 & - & - & 004 & - & - & 2,15 & - & - \\
\hline Galactia boavista (Vell.) Burkart & - & - & 002 & - & - & 2,12 & - & - \\
\hline Eupatorium betonicaeforme Baker & - & - & 006 & - & - & 2,08 & $4,30 \mathrm{~b}$ & - \\
\hline Piriqueta sp. & - & - & 004 & - & - & 2,00 & - & - \\
\hline Sebastiania hispida (Mart.) Pax ex Engl & - & - & 003 & - & - & 1,95 & - & - \\
\hline Commelina difusa Burm. f. & - & - & 004 & - & - & 1,72 & - & - \\
\hline Asteraceae sp. 4 & - & - & 005 & - & - & 1,70 & $2,55 b$ & - \\
\hline Lamiaceae sp1 & - & - & 007 & - & - & 1,55 & $7,00 \mathrm{~b}$ & - \\
\hline Vernonia cf. cataractarum Hieron & - & - & 003 & - & - & 1,55 & - & - \\
\hline Malpighiaceae & - & - & 003 & & & 1,52 & - & - \\
\hline Borreria poaya (A. St.-Hil.) DC & - & - & 003 & - & - & 1,39 & - & - \\
\hline Pavonia guerkeana R.E. Fr. & - & - & 002 & - & - & 1,33 & - & - \\
\hline $\begin{array}{l}\text { Chamaecrista desvauxii (Kunth ex Vogel) H. } \\
\text { S. Irwin \& Barneby }\end{array}$ & - & - & 002 & - & - & 1,30 & - & - \\
\hline Jacaranda oxyphylla Cham. & - & - & 002 & - & - & 1,28 & - & - \\
\hline Asteraceae sp. 1 & - & - & 002 & - & - & 1,27 & - & - \\
\hline Vernonia sp. 2 & - & - & 002 & - & - & 1,26 & - & - \\
\hline Asteraceae sp. 3 & - & - & 002 & - & - & 1,26 & - & - \\
\hline Asteraceae sp. 5 & - & - & 002 & - & - & 1,24 & - & - \\
\hline Eriosema heterophyllum Benth. & - & - & 002 & - & - & 1,24 & - & - \\
\hline Chamaecrista rotundifolia (Pers.) Greene ${ }^{b}$ & - & - & 002 & - & - & 1,24 & - & - \\
\hline Indeterminada 1 & - & - & 004 & - & - & 1,15 & - & - \\
\hline Rubiaceae sp. 1 & - & - & 004 & - & - & 1,09 & - & - \\
\hline Ascisanthera alsinaefolia (DC.) Triana & - & - & 003 & - & - & 0,91 & - & - \\
\hline Hyptis sp. & - & - & 001 & - & - & 0,68 & - & - \\
\hline Allagopteris campestris (Mart.) Kuntze & - & - & 001 & - & - & 0,66 & - & - \\
\hline Stylosanthes acuminata Ferreira \& S. Costa & - & - & 001 & - & - & 0,64 & - & - \\
\hline Eupatorium aff. kleinioides H.B.K. & - & - & 001 & - & - & 0,62 & - & - \\
\hline Indeterminada 2 & - & - & 001 & - & - & 0,62 & - & - \\
\hline Indeterminada 4 & - & - & 001 & - & - & 0,62 & - & - \\
\hline Indeterminada 5 & - & - & 001 & - & - & 0,62 & - & - \\
\hline Indeterminada 6 & - & - & 001 & - & - & 0,62 & - & - \\
\hline Stevia clauseni Schultz-Bip & - & - & 001 & - & - & 0,62 & - & - \\
\hline Camarea affinis A.St.-Hil. & - & - & 001 & - & - & 0,62 & - & - \\
\hline Mimosa micropteris (Burkart) Barneby & - & - & 001 & - & - & 0,16 & - & - \\
\hline
\end{tabular}

Em que: $\mathrm{NI}=$ número de indivíduos; $\mathrm{VI}=$ valor de importância; $\mathrm{CD}=$ Coeficiente de dispersão: a - padrão aleatório. b -padrão agregado (difere significativamente de 1,0 a $5 \%$ de probabilidade pelo teste T). GF = grupos fitogeográficos: 1 - Merdional; 2 - Centro-Sudeste; 3 - Centro-Oeste; 4 - Amazônia; 5 - Norte-Nordeste; 6 - sem registro. 
A baixa ocorrência de árvores associada a um menor porte da flora lenhosa no limite austral do Cerrado foi relatada para o Parque Estadual do Cerrado (UHLMANN et al., 1998), porém, nas restritas manchas de cerrado do PEG, esta característica mostrou-se ainda mais acentuada. Por exemplo, na área de estudo pode-se observar que os indivíduos de pequi (Caryocar brasiliensis) são subarbustos com altura máxima de $1,10 \mathrm{~m}$, diferindo de outras localidades de cerrado, com indivíduos apresentando porte arbóreo (UHLMANN et al., 1998; FIDELIS e GODOY, 2003). Esta característica pode estar relacionada com as geadas frequentes da região, que estariam restringindo o crescimento em altura de algumas espécies típicas. Tal hipótese é sustentada pelo estudo de Brando e Durigan (2005) que relataram a diminuição nas médias de altura, tanto para o estrato superior como para o inferior, na comunidade do cerrado da Estação Ecológica de Assis (SP), após forte geada.

Para a amostragem dos indivíduos com DAS menor que $3 \mathrm{~cm}$ (estrato médio), as dez espécies com os maiores valores de importância (VI) representaram 59,3\% do total, enquanto a soma dos valores relativos de dominância de cinco espécies foi de $50 \%$ (Tabela 1). Myrsine umbellata

TABELA 2: Dados estruturais de comunidades com amostragem de espécies lenhosas obtidos em estudos realizados em áreas de Cerrado.

TABLE 2: Structural data for plant communities with a sampling of woody species from studies conducted Cerrado areas.

\begin{tabular}{|c|c|c|c|c|c|c|c|}
\hline Referência & Localidade & Clima & Alt. (m) & $\begin{array}{l}\text { Critério de } \\
\text { Inclusão }\end{array}$ & $\mathrm{H}^{\prime}$ & N. spp & DT (ind/ha) \\
\hline Presente estudo & Tibagi-PR & $\mathrm{Cfb}$ & $900-1050$ & $\mathrm{DAS} \geq 3 \mathrm{~cm}$ & 3,09 & 47 & 2360,0 \\
\hline Uhlmann et al. (1998) & Jaguariaíva-PR & $\mathrm{Cfb}$ & $800-900$ & $\mathrm{DB}>3 \mathrm{~cm}$ & 2,79 & 33 & 1372,5 \\
\hline Durigan et al. (1994) & Itirapina-SP & Cwa & 800 & $\mathrm{~PB} \geq 15 \mathrm{~cm}$ & 3,08 & 44 & 2464,0 \\
\hline César et al. (1988) & Corumbataí-SP & Cwa & $800-830$ & $\mathrm{DB} \geq 3 \mathrm{~cm}$ & 3,64 & 101 & 9066,7 \\
\hline Araújo et al. (1999) & Franca-SP & $\mathrm{Cwb}$ & $810-870$ & $\mathrm{DA}_{(\mathrm{colo})} \geq 3 \mathrm{~cm}$ & 3,53 & 65 & 6566,0 \\
\hline Durigan et al. (2002) & Brotas-SP & Cwa & 710 & $\mathrm{DAJ}_{(50 \mathrm{~cm})} \geq 5 \mathrm{~cm}$ & 3,02 & 44 & 1150,0 \\
\hline Fidelis e Godoy (2003) & $\begin{array}{l}\text { S. Rita do P. } \\
\text { Quatro-SP }\end{array}$ & Cwag & $590-740$ & $\mathrm{PAS}>3 \mathrm{~cm}$ & 3,62 & 75 & 13976,0 \\
\hline Barreira et al. (2002) & Brasilândia-MG & Aw & 575 & $\mathrm{CAS}>9,5 \mathrm{~cm}$ & 2,95 & 62 & 1838,7 \\
\hline Neri et al. (2007) & $\begin{array}{l}\text { Sen. Modestino } \\
\text { Gonçalves-MG }\end{array}$ & $\mathrm{Cwb}$ & $887-949$ & $\mathrm{CAS} \geq 10 \mathrm{~cm}$ & 3,61 & 91 & 6476,7 \\
\hline Balduino et al. (2005) & Paraopeba-MG & - & $734-750$ & $\mathrm{CA}_{(30 \mathrm{~cm})} \geq 15,7 \mathrm{~cm}$ & 3,57 & 73 & 1990,0 \\
\hline Pinto et al. (2009) & $\begin{array}{l}\text { Cocalzinho de } \\
\text { Goiás-GO }\end{array}$ & - & 1200 & $\operatorname{DAS}_{(30 \mathrm{~cm})} \geq 5 \mathrm{~cm}$ & 4,45 & 65 & 912,0 \\
\hline Andrade et al. (2002) & Brasília-DF & - & $1048-1160$ & $\mathrm{DB}_{(30 \mathrm{~cm})}>5 \mathrm{~cm}$ & 3,53 & 63 & 1964,0 \\
\hline $\begin{array}{l}\text { Fonseca e Silva Junior } \\
\text { (2004) }\end{array}$ & Brasília-DF & $\begin{array}{l}\text { Aw- } \\
\text { Cwa }\end{array}$ & 1056 & $\mathrm{DB}_{(30 \mathrm{~cm})} \geq 5 \mathrm{~cm}$ & 3,16 & 53 & 1219,0 \\
\hline $\begin{array}{l}\text { Fonseca e Silva Junior } \\
\text { (2004) }\end{array}$ & Brasília-DF & $\begin{array}{l}\text { Aw- } \\
\text { Cwa }\end{array}$ & 1056 & $\mathrm{DB}_{(30 \mathrm{~cm})} \geq 5 \mathrm{~cm}$ & 3,40 & 54 & 970,0 \\
\hline Assunção e Felfeli (2004) & Brasília-DF & Cwa & $1000-1050$ & $\mathrm{DB}_{(30 \mathrm{~cm})} \geq 5 \mathrm{~cm}$ & 3,41 & 54 & 882,0 \\
\hline Felfili et al. (2002) & Água Boa-MT & $\mathrm{Aw}$ & $450-500$ & $\mathrm{DAS} \geq 5 \mathrm{~cm}$ & 3,69 & 80 & 995,0 \\
\hline $\begin{array}{l}\text { Nascimento e Saddi } \\
\text { (1992) }\end{array}$ & Cuiabá-MT & Aw & - & $\mathrm{DB}_{(10 \mathrm{~cm})} \geq 3 \mathrm{~cm}$ & 1,34 & 27 & 1686,0 \\
\hline $\begin{array}{l}\text { Nascimento e Saddi } \\
\text { (1992) }\end{array}$ & Cuiabá-MT & Aw & - & $\mathrm{DB}_{(10 \mathrm{~cm})} \geq 3 \mathrm{~cm}$ & 2,6 & 34 & 1978,0 \\
\hline Miranda (1993) & Santarém-PA & - & - & alt $\geq 1 \mathrm{~m}$ & - & 19 & 881,3 \\
\hline
\end{tabular}

Em que: $\mathrm{H}^{\prime}=$ índice de diversidade de Shannon; N. spp/família = número de espécie e de famílias; DT = densidade total; DAS = diâmetro a altura do solo; $\mathrm{DB}=$ diâmetro basal; $\mathrm{DA}_{\text {(colo) }}=$ diâmetro a altura do colo; DAJ = diâmetro a altura do joelho; $\mathrm{PAS}=$ perímetro a altura do solo; $\mathrm{PB}=$ perímetro basal; $\mathrm{CAS}=$ circunferência a altura do solo. 
obteve os maiores valores em densidade, frequência e dominância e, portanto, alcançou também a primeira posição em VI nesta classe amostral (Tabela 1). Além desta espécie, Miconia sellowiana e Alibertia concolor também obtiveram altos VI, mostrando a similaridade com o estrato superior.

$\mathrm{Na}$ análise das subparcelas (estrato inferior), apenas os indivíduos de Periandra mediterranea representaram $41 \%$ do total amostrado, sendo a espécie dominante neste estrato (Tabela 1). Esta alta densidade também foi encontrada nos campos com afloramentos rochosos presentes no PEG (CARMO, 2006), mostrando que a adaptação desta espécie aos fatores ambientais locais associados ao seu sucesso reprodutivo, está favorecendo a propagação de suas populações.

Nas espécies avaliadas nas áreas de cerrado do PEG, em que foram amostrados mais de cinco indivíduos (totalizando 50 espécies), em $82 \%$ o coeficiente de dispersão(CD) foi, significativamente, superior a 1 (Tabela 1), sugerindo uma distribuição com padrão agregado. Este resultado era esperado, pois a ocorrência frequente de moitas é uma característica notável da fisionomia das áreas do PEG.

Vários fatores podem levar à distribuição agregada, como a heterogeneidade ambiental, indicando sítios favoráveis para o estabelecimento das populações, e modo reprodutivo (LUDWIG e REYNOLDS, 1988). Durigan et al. (2002) sugeriram para o cerrado em Brotas - SP, que a alta agregação dos indivíduos está associada à facilidade na regeneração por brotação de estruturas subterrâneas, formando indivíduos geneticamente idênticos. Esta parece ser uma explicação plausível também para a área estudada, pois foi constatada a comunicação do sistema subterrâneo em muitos indivíduos amostrados, como por exemplo: Myrsine umbellata, Alibertia concolor, Myrceugenia alpigena, Miconia sellowiana, Calyptranthes concinna, Ilex sp1 e Periandra mediterranea.

Normalmente a regeneração por sistema subterrâneo se dá após ação do fogo sobre as espécies lenhosas, com consequente perda da parte aérea. Esta forte perturbação do ambiente pode ocasionar a quebra da dominância apical da estrutura caulinar, promovendo a emissão de vários ramos laterais ou a iniciação de gemas radiculares (APEZZATO-DAGLÓRIA, 2000), características estas vantajosas, pois promovem o rápido restabelecimento da cobertura vegetal. Contudo, isto possibilita a formação de novos indivíduos próximos à planta- mãe, aumentando assim a agregação dos indivíduos. Como a prática de queimadas era comum na região, inclusive com relatos para a área de estudo antes de se tornar uma Unidade de Conservação (IAP, 2002), sugere-se que a ação do fogo poderia ser uma possível explicação para a estrutura do cerrado do PEG, que além da forte agregação dos indivíduos, nota-se um investimento maior em emissão de ramos (com alta área basal) em relação à altura, pois $27 \%$ do total de plantas mostram-se perfilhadas.

A distribuição por grupos fitogeográficos de cerrados (RATTER et al., 2003) das espécies arbóreas e arbustivas que foram identificadas em nível específico amostradas neste estudo (totalizando 50 espécies, conforme Tabela 1), mostrou que $80 \%$ têm ocorrência na região meridional. Isto vem confirmar que cerrados paranaenses e paulistas têm maior similaridade como verificado por Ratter et al. (2003), tratando apenas o Parque Estadual do Cerrado como única área representante do Paraná. Foram encontradas ainda, $38 \%$ das espécies nas áreas que formam o grupo Centro-Sudeste, $18 \%$ no Centro-Oeste, $10 \%$ nas áreas disjuntas da Amazônia e 6 \% no Norte-Nordeste, confirmando um padrão fitogeográfico consistente na distribuição das espécies do bioma Cerrado.

Foram encontradas quatro espécies com ampla ocorrência de distribuição: Casearia sylvestris, Erythroxylum suberosum, Tabebuia aurea e Miconia albicans. Contudo, para $18 \%$ das espécies lenhosas relacionadas na Tabela 1, não houve citações para outras localidades de cerrado sensu stricto. Ratter et al. (2003), analisando 951 espécies de 376 áreas de cerrado, verificaram que aproximadamente $65 \%$ têm ocorrência rara; portanto, a presença de espécies exclusivas caracterizam as áreas de cerrados marginais, pelas variações ambientais próprias de cada localidade. Estudos realizados em regiões limítrofes de cerrado, como na Amazônia (RATTER et al., 2003) e na chapada do Araripe, nordeste brasileiro (COSTA et al., 2004), têm relatado a diminuição da riqueza florística típica deste bioma em relação à área nuclear, possivelmente, em consequência do isolamento geográfico e das condições climáticas distintas (COSTA et al., 2004). Esta característica também é evidente nos relictos de cerrado do Paraná, onde as geadas frequentes poderiam estar selecionando apenas espécies mais generalistas. Além disso, Hueck (1972) postulou que áreas marginais de cerrados, em virtude do clima, estariam sendo 
substituídas pela vegetação vizinha, permanecendo como ilhas dentro de um novo tipo de vegetação. Isto foi observado neste estudo, pois espécies abundantes encontradas nas áreas de cerrado, como Myrsine umbellata, Pera glabrata, Calyptranthes concinna, Copaifera langsdorffii, Casearia sylvestris e Alchornea triplinervia, são também as de maior densidade nas florestas dentro do parque (CARMO, 2006), o que confirma que pequenos relictos, como da área de estudo, são testemunhos da redução dos cerrados em consequência do avanço das florestas pelas condições climáticas atuais (MAACK, 1981).

\section{CONCLUSÃO}

As áreas de cerrado do PEG caracterizamse por uma menor estatura, riqueza e diversidade de sua flora, quando comparadas com os cerrados de outras regiões, possivelmente por estarem localizadas na zona limite de ocorrência do bioma, sob regime atual de um clima subtropical com influência direta de um clima temperado (mais úmido e frio), diferindo da região core do Cerrado (mais seco e quente). No entanto, mantêm a composição florística típica das savanas brasileiras, com presença de espécies características como, por exemplo, Ouratea spectabilis, Stryphnodendron adstringens e Caryocar brasiliense; porém, estas estão incluídas na lista vermelha de plantas ameaçadas de extinção do Estado do Paraná, por apresentarem distribuição em relictos e estes terem sido, em parte, devastados. Estas particularidades do cerrado, ao longo da Escarpa Devoniana no Estado do Paraná, evidenciam a importância da preservação e manejo do bioma no seu limite austral.

\section{AGRADECIMENTOS}

Os autores agradecem à Universidade Estadual de Ponta Grossa pelo apoio logístico e pela bolsa cedida à A. L. P. Andrade; ao CNPq pela concessão da bolsa do programa PIBIC-UEPG à G. A. da S. D. dos Santos; ao Instituto Ambiental do Paraná (IAP) pela permissão para execução do projeto.

\section{REFERÊNCIAS BIBLIOGRÁFICAS}

ANDRADE, L. A. Z.; FELFILI, J. M.; VIOLATTI,L. Fitossociologia de uma área de cerrado denso na RECOR-IBGE, Brasília-DF. Acta Botânica Brasílica, São Paulo, v. 16, n. 2, p. 225-240, abr. 2002.
ANGIOSPERM PHYLOGENY GROUP II. 2003. An update of the Angiosperm phylogeny group classification for the orders and families of flowering plants: APG II. Annals of the Botanical Journal of the Linnean Society, London, v. 141, n. 4, p. 399436, 2003.

APEZZATO-DA-GLÓRIA, B. Raízes gemíferas: uma abordagem anatômica e ecológica. In: CAVALCANTI, T. B. et al. (Org.). Tópicos atuais em Botânica: palestras convidadas do $51^{\circ}$ Congresso Nacional de Botânica. Brasília: Embrapa Recursos Genéticos e Biotecnologia/ Sociedade Botânica do Brasil, 2000. p. 73-77.

ARAÚJO, A. R. B.; TEIXEIRA, M. I. J. G.; RODRIGUES, R. R. Florística e fitossociologia de um trecho de cerrado no município de Franca. Naturalia, Rio Claro, v. 24, p. 153-170, 1999.

ASSUNÇÃO, S. L.; FELFILI, J. M. Fitossociologia de um fragmento de cerrado sensu stricto na APA do Paranoá, DF, Brasil. Acta Botânica Brasílica, São Paulo, v. 18, n. 4, p. 903-909, out./dez. 2004.

BALDUINO, A. P. do C. et al. fitossociologia e análise comparativa da composição florística do cerrado da flora de Paraopeba-MG. Revista Árvore, Viçosa, v.29, n. 1, p. 25-34, jan./fev. 2005.

BARREIRA, S. et al. Estudo da estrutura da regeneração natural e da vegetação adulta de um cerrado senso stricto para fins de manejo florestal. Scientia Forestalis, Piracicaba, n. 61, p. 64-78, junho 2002.

BASTOS, M. N. C. Levantamento florísico dos campos do estado Pará. I-Campo de Joanes (Ilha de Marajó) Boletim do Museu Paraense Emílio Goeldi, série Botânica, Belém, v. 1, n.1/2, p. 67-86, 1984.

BATALHA, M. A.; ARAGAKI, S.; MANTOVANI, W. Florística do cerrado em Emas (Pirassununga, SP). Boletim de Botânica da Universidade de São Paulo, São Paulo, v. 16, p. 49-64, 1997.

BATALHA, M. A.; MANTOVANI, W. Floristic composition of the cerrado in the Pé-De-Gigante Reserve (Santa Rita Do Passa Quatro, Southeastern Brazil) Acta Botânica Brasílica, São Paulo, v. 15, n. 3, p. 289-304, set./dez. 2001.

BATALHA, M. A.; MANTOVANI, W. Reprodutive phenological patterns of cerrado plant species at the Pé-de-Gigante reserve (Santa Rita do Passa Quatro, SP): a comparison between the herbaceous and woody floras. Revista Brasileira de Biologia, São Carlos, v. 60, n. 1, p. 129-145, mar. 2000.

BRANDO, P. M.; DURIGAN, G. Changes in cerrado vegetation after disturbance by frost (São 
Paulo State, Brazil). Plant Ecology, Perth, v. 175, n. 2, p. 205-215, Jan. 2005.

BROWER, J. E.; ZAR, J. H. Field and laboratory methods for general ecology. Dubuque: Wm. C. Brown Publishers, 1984. 226 p.

CARMO, M. R. B. Caracterização fitofisionômica do Parque Estadual do Guartelá, município de Tibagi, estado do Paraná. 2006. 142 f. Tese (Doutorado em Biologia Vegetal)-Universidade Estadual Paulista, Rio Claro, 2006.

CÉSAR, O. et al. Estrutura fitossociológica do estrato arbóreo de uma área de vegetação de cerrado no município de Corumbataí (Estado de São Paulo). Naturalia, Rio Claro, v. 13, p. 91-101, 1988.

COSTA, I. R. da; ARAÚJO, F. S.; LIMA-VERDE, L. W. Flora e aspectos auto-ecológicos de um encrave de cerrado na chapada do Araripe, Nordeste do Brasil. Acta Botânica Brasílica, São Paulo, v. 18, n. 4, p. 759-770, out./dez. 2004.

CRUZ, G. C. F. Alguns aspectos do clima dos Campos Gerais. In: MELO, M. S.; MORO, R. S.; Guimarães, G. B (Ed.). Patrimônio Natural dos Campos Gerais do Paraná. Ponta Grossa: Editora UEPG, 2007. Cap. 5, p. 59-72.

DIEDRICHS, L. A. O processo de criação do Parque Estadual do Guartelá. Monografia de Especialização. UFPR. Curitiba, 1995. 48 p.

DURIGAN, G. et al. Caracterização de dois estratos da vegetação em uma área de cerrado no Município de Brotas, SP. Acta Botânica Brasílica, São Paulo, v. 16, n. 3, p. 251-262, jul./set. 2002.

DURIGAN, G. et al. Inventário florístico do cerrado na estação ecológica de Assis, SP. Hoehnea, São Paulo, v. 26, n. 2, p. 149-172, dez. 1999.

DURIGAN, G.; LEITÃO FILHO, H. F.; RODRIGUES, R. R. Phytosociology and structure of a frequently burnt cerrado vegetation in SEBrazil. Flora, Jena, v. 189, n. 2, p. 153-160, 1994.

EGLER, W. A. Contribuições ao conhecimento dos campos da Amazônia. 1- Os campos do Ariramba. Boletim do Museu Paraense Emílio Goeldi, série Botânica, Belém, v. 4, p. 1-40, 1960.

EITEN, G. The cerrado vegetation of Brazil. The Botanical Review, New York, v. 38, p. 201-341, 1972.

FELFILI, J. M. et al. Composição florística e fitossociologia do cerrado sentido restrito no município de Água Boa - MT. Acta Botânica Brasílica, São Paulo, v. 16, n. 1, p. 103-112, jan. 2002.

FIDELIS, A. T.; GODOY, S. A. P. Estrutura de um cerrado stricto sensu na gleba cerrado Pé-de-
Gigante, Santa Rita do Passa Quatro, SP. Acta Botânica Brasílica, São Paulo, v. 17, n. 4, p. 531539, out./dez. 2003.

FONSECA, M. S.; SILVA JUNIOR, M. C. Fitossociologia e similaridade florística entre trechos de cerrado sentido restrito em interflúvio e em vale no Jardim Botânico de Brasília, DF. Acta Botânica Brasílica, São Paulo, v.18, n. 1, p. 19-29, jan./mar. 2004.

HUECK, K. As Florestas da América do Sul. São Paulo: Polígono, 1972. 465 p.

IAP-INSTITUTO AMBIENTAL DO PARANÁ. Plano de Manejo do Parque Estadual do Guartelá. Curitiba: IAP, 2002.

KLEIN, R. M.; HATSCHBACH, G. Fitofisionomia e notas complementares sobre o mapa fitogeográfico de Quero-Quero (Paraná). Boletim Paranaense de Geociências, Curitiba, v. 28-29, p. 159-188, 1970/1971.

LUDWIG, J. A.; REYNOLDS, J. F. Statistical ecology: a primer on methods and computing. New York: John Wiley e Sons, 1988. 337 p.

MAACK, R. Geografia física do Estado do Paraná. 2. ed. Rio de Janeiro: José Olympio Editora, 1981. $450 \mathrm{p}$.

MAACK, R. Notas complementares à apresentação preliminar do Mapa Fitogeográfico do Estado do Paraná (Brasil). Arquivos do Museu Paranaense, Curitiba, v. 7, p. 351-362, dez. 1949.

MANTOVANI, W.; MARTINS, F. R. Florística do cerrado na reserva biológica de Moji Guaçu, SP. Acta Botânica Brasílica, São Paulo, v. 7, n. 1, p. 33-60, jun. 1993.

MEIRA NETO, J. A. A.; SAPORETTI JÚNIOR, A. W. Parâmetros fitossociológicos de um cerrado no Parque Nacional da Serra do Cipó, MG. Revista Árvore, Viçosa, v. 26, n. 5, p. 645-648, set./out. 2002.

MELO, M. S. Canyon do Guartelá, PR - Profunda garganta fluvial com notáveis exposições de arenitos devonianos. In: SCHOBBENHAUS, C. et al. (Ed.) Sítios Geológicos e Paleontológicos do Brasil. 1. ed. Brasilia: DNPM/CPRM - Comissão Brasileira de Sítios Geológicos e Paleobiológicos (SIGEP), 2002, v. 01, p. 279-288.

MIRANDA, I. S. Estrutura do estrato arbóreo do cerrado amazônico em Alter-do-Chão, Pará, Brasil. Revista Brasileira de Botânica, São Paulo, v.16, n. 2, p. 143-150, jun. 1993.

MUELLER-DOMBOIS, D.; ELLENBERG, $\mathrm{H}$. Aims and methods of vegetation ecology. New York: John Wiley e Sons, 1974. 547 p. 
NASCIMENTO, M. T.; SADDI, N. Structure and floristic composition in an area of cerrado in CuiabáMT, Brazil. Revista Brasileira de Botânica, São Paulo, v. 15, n. 1, p. 47-55, mar. 1992.

NERI, A. V. et al. Análise da estrutura de uma comunidade lenhosa em área de cerrado sensu stricto no Município de Senador Modestino Gonçalves, Norte de Minas Gerais, Brasil. Revista Árvore, Viçosa, v. 31, n. 1, p. 123-134, jan./fev. 2007.

PARANÁ. Secretaria de Estado do Meio Ambiente. Lista vermelha de plantas ameaçadas de extinção no estado do Paraná. Curitiba: SEMA/ GTZ, 1995. 139 p.

PASSOS, M. M. Biogeografia e Paisagem. 2. ed. Maringá: [s. n.], 2003. 264 p.

PINTO, J. R. R.; LENZA, E.; PINTO, A. S. Composição florística e estrutura da vegetação arbustivo-arbórea em cerrado rupestre, Cocalzinho de Goiás, Goiás. Revista Brasileira de Botânica, São Paulo, v. 32, n. 1, p. 1-10, jan./mar. 2009.

RATTER, J. A.; BRIDGEWATER, S.; RIBEIRO, J. F. Analysis of the floristic composition of the brazilian cerrado vegetation III: comparison of the woody vegetation of 376 areas. Edinburgh Journal of Botany, Edinburgh, v. 60, n. 1, p. 57 109, Mar. 2003.

RATTER, J. A.; RIBEIRO, J. F.; BRIDGEWATER, $\mathrm{S}$. The Brazilian Cerrado vegetation and threats to its biodiversity. Annals of Botany, Exeter, v. 80, n. 3, p. 223-230, Sept. 1997.

RIBEIRO, J. F.; WALTER, B. M. T. Fitofisionomias do Bioma Cerrado. In: SANO, S.; ALMEIDA, S. P. (Ed). Cerrado: ambiente e flora. Brasília: Embrapa Cerrados, 1998. p. 87-166.

SANAIOTTI, T. M.; BRIDGEWATER, S.; RATTER, J. A. A floristic study of the savanna vegetation of the state of Amapá, Brazil, and suggestions for its conservation. Boletim do Museu Paraense Emílio Goeldi, série Botânica, Belém, v. 13, n. 1, p. 1-27, 1997.

SHEPHERD, G. J. Fitopac 1. Manual de Usuário. Campinas: Departamento de Botânica, UNICAMP, 1995.

SILVA, L. O et al. Levantamento florístico e fitossociológico em duas áreas cerrado sensu stricto no Parque estadual da Serra de Caldas Novas, Goiás. Acta Botânica Brasílica, São Paulo, v. 16, n. 1, p. 43-53, jan. 2002.

TAKEDA, I. J. M.; MORO, R. S.; KACZMARECH, $\mathrm{R}$. Análise florística de um encrave de cerrado no Parque do Guartelá, Tibagi, PR. Publicatio, Ponta Grossa, v. 2, n. 1, p. 21-31, 1996.

TOREZAN, J. M. D. Nota sobre a vegetação do rio Tibagi. In: MEDRI, M. E. et al. (Ed.). A Bacia do Rio Tibagi. Londrina: UEL/Copati/Klabin, 2002. Cap. 7, p. 103-107.

UHLMANN, A. et al. Relações entre a distribuição de categorias fitofisionômicas e padrões geomórficos e pedológicos em uma área de savana (cerrado) no estado do Paraná, Brasil. Arquivos de Biologia e Tecnologia, Curitiba, v. 40, n. 2, p. 473483, jun. 1997.

UHLMANN, A.; GALVÃO, F.; SILVA, S. M. Análise da estrutura de duas unidades fitofisionômicas de savana (cerrado) no sul do Brasil. Acta Botânica Brasílica, São Paulo, v. 12, n. 3, p. 231-247, dez. 1998.

VELOSO, H. P.; RANGEL FILHO, A. L. R; LIMA, J. C. A. Classificação da vegetação brasileira, adaptada a um sistema universal. Rio de Janeiro: IBGE - DERMA, 1991. $123 \mathrm{p}$.

VON LINSINGEN, L. et al. Composição florística do Parque Estadual do Cerrado de Jaguariaíva, Paraná, Brasil. Acta Biológica Paranaense, Curitiba, v. 35, n. 3-4, p. 197-232, 2006. 